\title{
Propuesta de intervención didáctica para la enseñanza-aprendizaje del léxico en español como segunda lengua. Adaptación curricular en la enseñanza primaria
}

\author{
Cristina del Moral Barrigüete \\ Facultad de Ciencias Sociales y Humanas \\ Teruel
}

Recibido: 5 de junio de 2011 / Aceptado: 23 de diciembre de 2011

ISNN: $1697-7467$

\begin{abstract}
RESUMEN: El punto de partida de este trabajo es el diseño y puesta en práctica de una investigación sobre el léxico disponible, cuya metodología se advierte cuasiexperimental, llevada a cabo en un grupo concreto de alumnos de la Ciudad Autónoma de Melilla que no tienen el español como lengua materna y que cursan el último curso $\left(6 .^{\circ}\right)$ de la Educación Primaria en dicha localidad. Esta propuesta didáctica se considera como prospectiva también de utilidad para la labor docente universitaria, como formadores de futuros maestros con los que se trabaja también el desarrollo de esta competencia y sus aplicaciones didácticas.

Palabras clave: enseñanza-aprendizaje, léxico, didáctica, español, segundas lenguas
\end{abstract}

Educational Intervention Proposal for Teaching and Learning Vocabulary in Spanish as a Second Language. Syllabus adjustment for Primary Education

\begin{abstract}
The starting point of this paper is the design and implementation of an cuasi-experimental research on the lexicon available of a group of students who do not have Spanish as their mother tongue, in spite of being residents in Melilla and that they attend the last academic year of primary education $\left(6 .^{\circ}\right)$. This research is also useful for our university teaching, because we are trainers of future teachers and work with them also the development of this competence and their didactic applications.

Keywords: teaching and learning, vocabulary, teaching, Spanish, second language
\end{abstract}

\section{INTRODUCCIÓN}

Muchos autores han señalado pautas acerca del proceso requerido para aprender una palabra nueva en una L2; dicho proceso implica conocer muchos aspectos relacionados con ella, sin los cuales la adquisición de esta sería parcial e incompleta (Martín, 2004: 23). Así, siguiendo a Jack C. Richards (1991), para aprender una palabra no basta con conocer su significado, tenemos que conocer el grado de probabilidad de encontrarla en el lenguaje oral y escrito; tener presentes sus limitaciones de uso temporal, geográfico, social y discursivo; dominar su comportamiento sintáctico, su forma, sus posibles derivadas y las redes asociativas con otras palabras. 
Por otro lado, sabemos que las palabras más disponibles son aquellas que se recuperan del lexicón mental en primer lugar y que, por tanto, aparecen en los puestos iniciales de cualquier lista de palabras. Lógicamente, no siempre hay coincidencia en el índice de disponibilidad que hablantes distintos otorgan a un mismo término. Para establecer la media de los resultados es preciso recurrir al criterio de frecuencia: se ha de ponderar la posición de los vocablos en una lista dada con la frecuencia de aparición de los mismos.

No cabe ninguna duda entonces de que para aprender una nueva palabra no basta con leerla una o dos veces, o con buscarla en el diccionario y copiar su significado: es necesario un proceso de adquisición de cierta complejidad, estructurado en varias fases para que el nuevo vocablo pase a formar parte del lexicón mental del alumno como L2 (Martín, 1999: 159-160): (i) la entrada (una media de 8 a 12 en una clase de una hora); ii) el almacenamiento (el lexicón mental está muy organizado y es eficiente); y iii) la recuperación de la información (muchas veces no funciona, bien por la teoría del olvido: porque la información no se activa regularmente, o bien porque somos incapaces de recuperarla).

Nuestro panorama didáctico presenta la peculiaridad de estar especialmente adaptado a un contexto muy particular, como es el caso de los colegios públicos de Melilla, donde están escolarizados gran número de alumnos de origen magrebí que no tienen como lengua materna el castellano, y que lo dominan en distintos grados dependiendo del nivel de influencia de la gran cantidad de factores sociales, económicos, políticos y culturales que configuran la compleja realidad de esta ciudad. Nuestra investigación se orienta así al logro de un objetivo fundamental como es el grado de repercusión en la competencia léxica en español como L2 de estos alumnos. Así, proponemos a continuación una propuesta didáctica para la innovación en el terreno de la enseñanza primaria en contextos multiculturales en el que los aprendizajes tradicionales, basados fundamentalmente en la memorización de piezas léxicas, se han revelado, tanto en el plano de la práctica como en las consecuencias didácticas derivadas de las aportaciones de las nuevas ciencias del lenguaje (Oster, 2005; San Mateo, 2005; Vivanco, 2001), como insuficientes, cuando no francamente ineficaces. Para poder romper la inercia de las pautas habituales lo principal es -a nuestro juicio- ofrecer al profesorado experiencias y materiales empíricamente probados para orientar así su quehacer hacia un cambio que le lleve a introducir en sus programaciones, de forma sistemática e integrada con el resto de los contenidos y objetivos del área, los relativos al dominio léxico.

Nuestra propuesta es una "adaptación" del método Collage (Möller, J. y Burbat, R., 2001 y 2007), elaborado en su origen para la enseñanza del alemán como lengua C (o segunda lengua extranjera) en la Facultad de Traducción e Interpretación de la Universidad de Granada, confeccionado posteriormente para la enseñanza del español ${ }^{1}$, con especial atención al léxico en el estudio de segundas lenguas y contextualizado finalmente en el entorno particular de convivencia de lenguas que se da en la Ciudad Autónoma de Melilla.

Si bien, tenemos que anotar a nuestro favor que los alumnos investigados con dificultades de comprensión idiomática por no ser hablantes nativos, no estaban mezclados en un

\footnotetext{
${ }^{1}$ Proyecto de innovación docente (2004-06) "Método Collage: Enseñanza de una segunda lengua extranjera en la formación del traductor/intérprete", coordinado por Julia Möller Runge (profesora titular de la Universidad de Granada), y proyecto en el que se estudiaron las peculiaridades de la enseñanza del español como segunda lengua y se analizaron las posibilidades de adaptación de Collage determinando, en su caso, posibles cambios y adaptaciones para el español en comparación con el alemán.
} 
contexto escolar con hablantes nativos y, por tanto, podría haberse incluido en el Proyecto de Centro un programa diseñado especialmente para ellos por ser un colegio de la periferia de la ciudad, con un $100 \%$ de alumnos con lengua materna chelja (dialecto árabe hablado en la zona del Rif, Marruecos), y no el mismo programa que para los otros hablantes nativos de la ciudad, como apuntaremos más adelante cuando hablemos de los materiales utilizados.

La hipótesis central de nuestra investigación para este afanoso estudio, relacionado con la comparación entre dos grupos de alumnos (experimental y control) antes y después de ser sometidos a un programa específico de aprendizaje léxico, quedaría formulada de la siguiente manera:

Los alumnos de sexto curso del Tercer Ciclo de la Educación Primaria con lengua materna diferente al español que siguen el Programa Didáctico para el Desarrollo de la Competencia Léxica (Pedelex) mejoran su léxico disponible, en mayor proporción que aquellos alumnos que no siguen este método.

\section{Material Y MÉTOdos}

Con los objetivos y las hipótesis planteados, procede ahora determinar cómo se ha realizado el diseño de la investigación empírica atendiendo a los tres elementos principales que en este apartado se examinan, a saber: participantes, material empleado y procedimiento.

Consideramos que el tipo de investigación que más se ajusta al carácter de este trabajo es el descriptivo, así como el inferencial para el análisis de los resultados obtenidos. Por otra parte, de los diferentes diseños de investigación existentes -transversal, longitudinal y secuencial- se ha escogido el transversal, por realizar la recogida de datos a lo largo de un periodo de tiempo determinado para procesar y analizar con posterioridad los datos obtenidos. Si bien, en el campo de la Adquisición de Segundas Lenguas (ASL) existen otras referencias a la implementación de estudios longitudinales con la finalidad de comprobar los efectos de diversos factores en dicha adquisición (Ortega \& Iberri-Shea, 2005; Singleton \& Ryan, 2004). Por último, el estudio empírico de nuestra investigación parte de un diseño que Dendaluce (1989) denomina cuasiexperimental, pues la muestra resulta seleccionada solo relativamente por el azar, en tanto en cuanto se trabaja con grupos y niveles previamente establecidos por el centro donde se lleva a cabo dicho estudio empírico.

\subsection{Participantes}

Hemos utilizado muestras representativas de la población a la que pretende ir dirigida la investigación, como hemos dicho, alumnos de la Educación Primaria obligatoria que tienen el español como segunda lengua (L2). Para la tipificación, la muestra es de un centro público de la periferia de Melilla, donde la población es en un $100 \%$ de habla materna chelja. La muestra total es de 55 sujetos, de los que 25 eran varones y 30 , mujeres. Los participantes previstos son dos grupos correspondientes al $6 .^{\circ}$ nivel de Educación Primaria del Centro de Educación Infantil y Primaria (CEIP) "Constitución", de la Ciudad Autónoma de Melilla. 
y con un dominio del español en distintos grados que depende del nivel de influencia de la gran cantidad de factores sociales, económicos, políticos y culturales que configuran la compleja realidad de esta ciudad y, en concreto, la de estos alumnos. Resumimos aquí las siguientes características socioculturales de los mismos:

Casi la totalidad de los niños, el $90 \%$ de los sujetos son nacidos en Melilla y el $85,5 \%$ han vivido siempre allí. En cuanto a la relación que tienen los sujetos con Marruecos, constatamos en el estudio que realizamos que la opción elegida por un $83,6 \%$ del total de sujetos es que van con frecuencia (fines de semana, vacaciones, etc.). Como era esperable, en torno al 50\% de los padres de los sujetos han nacido en Marruecos y otro tanto en Melilla. En cuanto a los datos sobre los estudios del padre y de la madre, destacamos que una parte importante de los sujetos $(34,5 \%)$ no sabe o no contesta, creemos que debido a que hay muchos que sólo cumplimentan el ítem de los estudios del padre pues en la cultura árabe es habitual que las madres no tengan estudios y tampoco trabajen fuera de casa. Así, en más de la mitad de los casos $(52,7 \%)$ la madre no tiene estudios y sólo un $12,7 \%$ tiene estudios primarios.

Casi en su totalidad $(96,4 \%)$ no son repetidores. No obstante, llama la atención que el $36,4 \%$ de los sujetos obtuvo un 3 (insuficiente) en la nota de Lengua Española del curso anterior. Es curioso, además que la mitad de los sujetos (60\%) se reconoce como hablantes de español y chelja. Y casi un $70 \%$ reconoce que utiliza las dos lenguas en casa y fuera del colegio.

Otras preguntas relacionadas con el contexto sociocultural en el que se desenvuelven estos sujetos era no sólo saber si los niños veían la televisión, sino cuántas horas permanecían delante de la pantalla. El $70 \%$ de los sujetos ve la televisión española entre una y tres horas diarias.

En relación con el gusto por la lectura -incluida esta cuestión por la repercusión que puede tener en el grado de dominio lingüístico- llama la atención que los sujetos no niegan su falta de interés por esta actividad, aunque normalmente intuimos que los niños saben lo que tienen que decir en estos casos y, aunque no sea verdad, marcan la opción que según ellos se espera. La gran mayoría (el 70\%) dice que lee libros. Sólo el 10,9\% especificaron con exactitud qué clase de libros (cómics y/o cuentos), pero en general no podemos fiarnos del todo de estos datos, por lo que hemos dicho ya.

La muestra de la investigación ha estado formada, como se ha dicho ya, por dos grupos de sujetos: un grupo experimental, formado por 27 alumnos del curso $6 .{ }^{\circ} \mathrm{B}$, tercer ciclo de Educación Primaria (EP), y con el que se ha desarrollado todo el programa elaborado por nosotros, Pedelex; y otro grupo control, formado por 28 alumnos del curso $6 .{ }^{\circ} \mathrm{A}$, tercer ciclo de la Educación Primaria, con el que no se ha desarrollado ninguna actividad relacionada con nuestro programa y se ha limitado a seguir -para todos los aspectos incluidos en el área de Lengua Castellana y Literatura- las pautas que marca el libro de texto que se utiliza en el centro, el de la editorial Anaya (VV.AA., 2006)².

\footnotetext{
${ }^{2}$ Las razones por las que el Centro ha escogido un libro de aprendizaje del español como L1 creemos que son debidas a que en esta zona geográfica predomina la lengua española como dominante frente al chelja.
} 


\subsection{Material empleado}

Hemos considerado en nuestra investigación dos tipos de variables:

a) Una variable dependiente (VD) sobre el léxico disponible para el estudio intergrupos que compara los resultados obtenidos por el grupo experimental y por el grupo control. Esta variable es, a su vez, objetiva, ya que expresa lo que el alumno es capaz de completar en la prueba realizada.

b) Una variable independiente (VI) correspondiente a un programa que sigue, entre otros, los principios del método Collage para el desarrollo de la competencia léxica, y que no presentamos aquí por no exceder los límites de este trabajo.

La prueba del pre-test trata de comprobar, a grandes rasgos, el nivel de partida del alumnado sobre el léxico disponible. Su utilización en el aula fue justificada ante los estudiantes, sobre todo, por el interés que tenía para ellos la toma de conciencia de sus carencias y de sus logros. De ahí que, una vez revisadas y evaluadas tanto por las profesoras de cada grupo como por el investigador ${ }^{3}$, se devolvieran a los alumnos para corregirlas entre todos. De este modo, no sólo se comprueban errores y aciertos, sino que también se repasan contenidos básicos -especialmente procedimentales- del área de Lengua Castellana y Literatura durante los primeros días del curso.

La prueba final -o del post-test- se elaboró para ser aplicada al término del programa de intervención para el desarrollo de la competencia léxica. Se ha procurado que sea lo más parecida posible a las inicial para comprobar si ha habido evolución en los diferentes campos semánticos del léxico disponible que se evaluaban.

Para la evaluación del léxico disponible se ha realizado una tarea de compendio de palabras relacionadas con dieciséis campos semánticos, adoptando como elemento estructural los llamados "centros de interés", fijados por Gougenheim en 1956, y aplicados a la enseñanza de la lengua francesa ${ }^{4}$. Los catálogos estadísticos basados en el criterio de disponibilidad léxica toman como punto de partida los 16 centros de interés con los que se trabaja en la actualidad, y que ofrecen un doble interés: por un lado, actúan como desencadenantes de los vocablos que presentan un mayor índice de disponibilidad; por otro, dan cuenta de la organización temática latente en el proceso de asimilación léxica. Estos centros de interés son perfectamente válidos para nuestro contexto de investigación, si bien la clasificación propuesta es esencialmente orientativa y está abierta a la incorporación de nuevos marcos

${ }^{3}$ Para la investigación, las puntuaciones que se han considerado han sido las nuestras.

${ }^{4}$ El nacimiento de estas investigaciones está directamente relacionado con la intención de facilitar la adquisición de la lengua francesa, tanto a los habitantes de los países africanos que habían formado parte de la Unión Française, como al importante número de inmigrantes que en esos momentos entraba a formar parte de la población de Francia. Gougnheim y sus colaboradores se propusieron crear una "lengua de base", limitada a sus unidades esenciales en el vocabulario y en la gramática, con el fin de garantizar una adquisición rápida del francés. Ese proyecto de simplificación del francés para la enseñanza a extranjeros se materializó en 1956 con la aparición de Le français élémentaire y, posteriormente, autores como López Morales (1999) han utilizado esos dieciséis centros de interés o campos semánticos como referente para el aprendizaje del español. 
temáticos, si así lo requieren las necesidades de adaptación a ciertas realidades culturales (Samper, 2002: 21-22). Los dieciséis campos considerados en esta investigación actúan como estímulo en la activación espontánea del vocabulario: dado un centro de interés, el encuestado ha de indicar cuántos vocablos relacionados con el tema acudan a su mente en un periodo de tiempo limitado.

Realizamos una investigación cuyo objeto es el estudio de la producción discursiva escrita de escolares de primaria, con el objetivo de caracterizar su actuación léxica en el sexto nivel del tercer ciclo de esta etapa. Nos valemos para ello de determinadas variables extralingüísticas y de nuevos criterios, propios de los estudios de léxico-estadística y de la lingüística de corpus (Pérez, 2002; Sinclair, 2004), que permiten precisar la relevancia de las palabras que forman parte del corpus estudiado.

En este estudio consideramos como indicadores de la actuación léxica, entre otros, la "amplitud y dominio del vocabulario", y la "riqueza léxica", manifestados en el uso de las estrategias generales y específicas que se detallan en los instrumentos utilizados en la investigación. El primer indicador constituye una manifestación del conocimiento que el hablante tiene de la realidad que le rodea, y se relaciona con la cantidad, crecimiento y calidad del vocabulario empleado. El segundo indicador se define a partir del número de vocablos nuevos que figuran en un texto dado (Müller, 1977: 116). Es decir, según aumente el número de vocablos, en correspondencia con el crecimiento del texto, también irá creciendo el índice de riqueza léxica, aunque no al mismo ritmo.

Por todo ello, no sólo valoramos la competencia léxica cuantitativamente, sino que también vemos de vital importancia valorar la riqueza léxica y el conocimiento de estrategias para usar las palabras de forma eficaz y adecuada en cualquier contexto de comunicación (Cottez, 1987: 276, y citada por Prado, 2004: 309).

Las pruebas que usamos son de aplicación individual, y provienen de las utilizadas para el léxico disponible de Melilla (Fernández et al., 2008: 156-161). Al tratarse de niños, creímos conveniente realizar una lluvia de ideas de las palabras que se les ocurrían a los sujetos de cada centro de interés. Controlamos el tiempo, el doble de lo que necesitaría un nativo, según lo establecido, para rescatar las 20 primeras palabras que se les vinieran a la mente. Otra variante de esta lluvia de ideas, realizada en el post-test, fue jugar a una "versión casera" del Scattergoris ${ }^{5}$. Esta versión consiste en decir una letra y anotar una palabra de cada centro de interés que empiece por ella. El primero en terminar todos los centros de interés con la letra propuesta, es el ganador. La duración de esta actividad variará dependiendo de la agilidad de los niños a la hora de completar los dieciséis centros de interés, según las letras propuestas en cada "tirada".

El libro de texto de Lengua Castellana y Literatura de Educación Primaria utilizado en el centro (Anaya, 2006) y el nivel donde se ha llevado a cabo la investigación se ha tomado como referencia para insertar de forma significativa lo trabajado en el programa con lo dispuesto para el curso, integrando, en lo posible, ambos núcleos. En este manual trata, sobre todo, el mundo animal y la naturaleza, dejando atrás otros centros de interés tan

${ }^{5}$ En el juego del Scattergoris se elige una de las opciones de preguntas sobre comidas, ríos, nombres propios, etc. y se tira un dado de letras, se pone el tiempo (máx. 1 min.) y se tiene que escribir sobre ese campo y por la letra que ha salido. 
importantes para el niño como la alimentación, el cuerpo humano, la familia, el clima, la ciencia, la electricidad, etc. Por otro lado, algunos de los temas corresponden con el contenido de las lecturas adaptadas con que comienza cada unidad. Se puede observar, a simple vista, que no hay ninguna relación entre el tema y el vocabulario que debemos enseñar, lo cual nos parece ciertamente un error, pues al final, los contenidos de vocabulario siguen estando descontextualizados y el niño aprende palabras fuera de lugar.

No obstante, tenemos que admitir que en este manual se trabaja bastante el vocabulario en cada una de las secciones del libro: Expresión escrita, Ortografía, Gramática y Expresión oral. Nos han llamado la atención en especial tres secciones más que ayudan enormemente al aprendizaje del vocabulario contextualizado $-\mathrm{y}$ que no suelen aparecer en otros manuales- y son: Trabaja lo aprendido, Juega con el lenguaje y Vuelve atrás. Especialmente en el apartado Juega con el lenguaje aparecen chistes, adivinanzas, poemas, crucigramas, etc., que ayudan al alumno a memorizar palabras o expresiones nuevas y a revisar las ya conocidas.

En cuanto a los materiales que integran nuestra propuesta, estos son un cuadernillo de tareas para cada unidad y dos fichas de evaluación (una de desarrollo y otra final) también para cada unidad. En total, hemos elaborado cinco unidades para este trabajo, como muestra que juzgamos suficiente para comprobar el éxito del programa. Cada unidad consta de entre 5 y 10 tareas y comienza siempre con una clasificación de palabras según el campo semántico que estemos trabajando. La siguiente actividad es especificar el vocabulario que desconocen y aclararlo. Después continuamos normalmente con un texto y preguntas de comprensión lectora, a la vez que vamos haciendo preguntas sobre el tipo de texto, el emisor-receptor, para ir progresando en la lengua española, etc. Finalmente terminamos la unidad siempre con humor, explicando algunos chistes relacionados con el vocabulario de la unidad, en los que se origina la opinión.

\subsection{Procedimiento}

Por supuesto, el anonimato de los sujetos participantes es un principio ético inherente a la investigación y, sobre todo, obligado en el campo de la educación. De acuerdo con este principio, se ha utilizado una combinación de metodología cuantitativa y cualitativa; es decir, se aportan los datos cualitativos de la experiencia proporcionados por el profesorado en sesiones de puesta en común y, desde un punto de vista cuantitativo, los resultados de las pruebas se analizan estadísticamente para proceder a su interpretación y tratar de obtener así una visión lo más completa posible del fenómeno estudiado, a fin de comprobar hasta qué punto se cumplen nuestros objetivos y nuestras hipótesis de partida.

Nuestra investigación ha tenido tres fases complementarias claramente diferenciadas:

Primera fase. En la que se han llevado a cabo dos acciones:

a) Hemos realizado un modelo de programa para el desarrollo de la competencia léxica (Pedelex), cuya aplicación constituye la variable independiente de la investigación. El modelo se enmarca, como hemos dicho ya, dentro de las propuestas innovadoras del método Collage y su concreción en el ámbito del léxico y de su enseñanzaaprendizaje, donde priman las diferentes estrategias, técnicas y procedimientos necesarios para adquirir nuevas palabras y asentar las ya conocidas.

b) Se han realizado las pruebas referidas a la variable dependiente, como pre-test para conocer la situación de partida de los sujetos que componen la muestra, tanto del grupo experimental como del grupo control. 
Segunda fase. En esta segunda fase han tenido lugar tres acciones:

a) La aplicación del programa, desarrollando las sesiones establecidas por la dirección del colegio, dos sesiones a la semana (lunes y viernes) de cincuenta minutos.

b) La revisión y evaluación del desarrollo del programa de la variable independiente siguiendo las categorías y subcategorías de la hoja de observación de la competencia léxica.

c) La realización de las pruebas referidas a las variables dependientes como post-test para comprobar los resultados del programa.

Tercera fase. En esta tercera fase se han efectuado dos acciones más:

a) La elaboración, análisis y discusión de los resultados obtenidos.

b) Finalmente, la redacción del informe final que constituye el presente trabajo.

En el estudio empírico hemos utilizado el análisis descriptivo e inferencial de dos variables. Dado, además, el volumen del tratamiento estadístico realizado hemos utilizado programas con tratamiento informático, en concreto, el SPSS (Statistical Package for the Social Sciences) para Windows en su Versión 15 (2006).

Hasta llegar a la obtención de los resultados finales, hemos seguido diferentes tipos de estudios de los datos, a saber:

- Análisis de fiabilidad, para comprobar si hay una buena correlación inter-elementos entre las variables dependientes del estudio intergrupos. Entre otras pruebas se han realizado el Alfa de Cronbach, la Correlación Item-Total, el equivalente del ANOVA con la prueba de Friedman, la prueba de no aditividad de Tukey y el coeficiente de concordancia $W$ de Kendall.

- Análisis descriptivo exploratorio de las variables medidas en los sujetos mediante la estadística descriptiva univariable, es decir, se ha tratado de comprender cada una de las variables independientemente, conociendo sus medidas de tendencia central, o media, mediana, desviación típica, asimetría y curtosis, fundamentalmente.

\section{Resultados}

A continuación ofrecemos los resultados obtenidos tanto en los estudios estadísticos descriptivos sobre el léxico disponible de los grupos experimental y control como en las pruebas no paramétricas de $U$ de Mann Whitney, es decir, los estadísticos de contraste de las pruebas del pre-test y del post-test.

Tenemos que advertir además que aunque no estamos analizando la utilidad de las pruebas de nuestra investigación, puesto que se trata de pruebas estandarizadas, hicimos un test de fiabilidad de todas las variables consideradas, dando los resultados siguientes: 52 casos válidos $(94,5 \%), 3$ excluidos $^{6}(5,5 \%)$, del total conformado por 55 casos $(100 \%)$. En nuestro

${ }^{6}$ Los valores excluidos lo están porque se separan mucho de la media, es decir, algunos no hicieron todas las pruebas por diversos motivos como, por ejemplo, ausencia a clase ese día por enfermedad, asistencia al aula de Pedagogía Terapéutica (PT). 
caso, el Alfa de Cronbach nos dice que los elementos tipificados en esta variable tienen una alta correlación, 0,916, por lo que estadísticamente es muy significativo o están muy relacionados. Por otra parte, la Correlación Item-Total es de gran relevancia como muestran la siguientes tablas (Tablas I y II) para corroborar la significatividad o ausencia de ésta.

Las variables sobre el léxico disponible que mayor correlación inter-elementos obtuvieron en nuestro caso fueron las distribuidas en los campos semánticos sobre "ropa", "partes de la casa", "alimentos y bebidas" y "animales". Por todo ello, podemos decir que, en general, hay una buena correlación inter-elementos en nuestra investigación sobre léxico disponible en el pre-test.

En cuanto a los estadísticos de fiabilidad referidos al léxico disponible post-test hubo un $98 \%$ de casos válidos (52), tan solo un 1,9\% de casos excluidos de un total de 53 casos. Los valores excluidos se han acortado con respecto a la prueba inicial sobre léxico disponible, es decir, hay menos casos que están muy separados de la media. El Alfa de Cronbach en el léxico disponible post-test basada en los dieciséis elementos tipificados es también altamente significativo al 0,917 . Y la matriz de correlaciones inter-elementos en el léxico disponible post-test ha disminuido considerablemente respecto al pre-test. Tan sólo el campo de la "ropa" y las "partes de la casa" conserva aún cierta validez estadística. Esto quiere decir que el valor obtenido no tiene significatividad estadística, con lo que, nuestro grupo experimental, aun teniendo un nivel inicialmente más bajo, ha conseguido igualarse con el grupo control. 


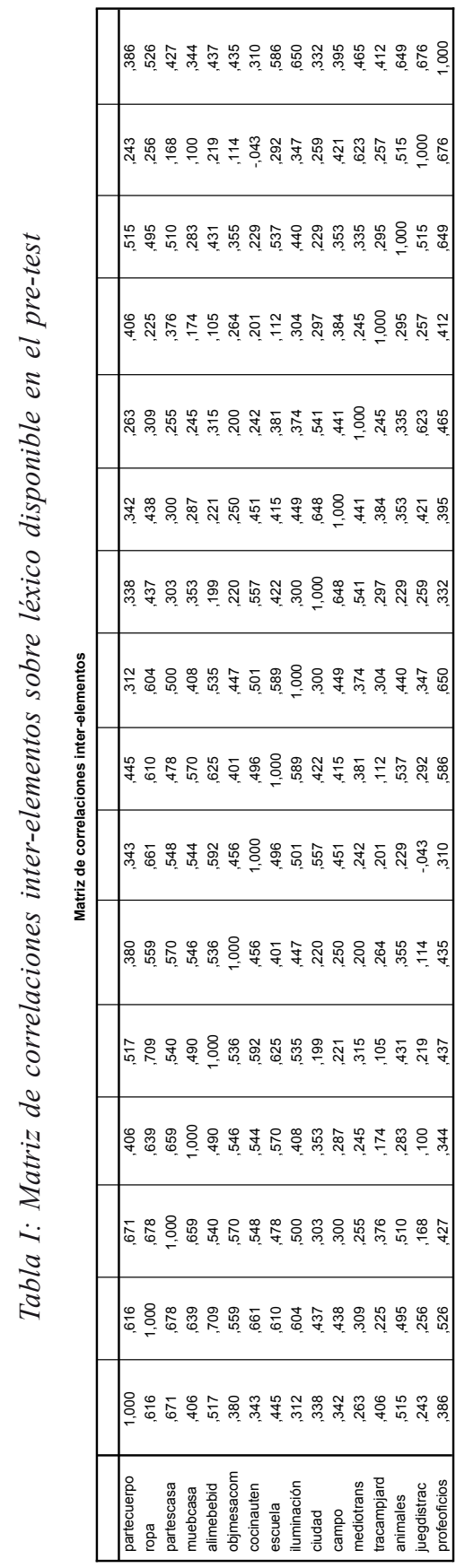

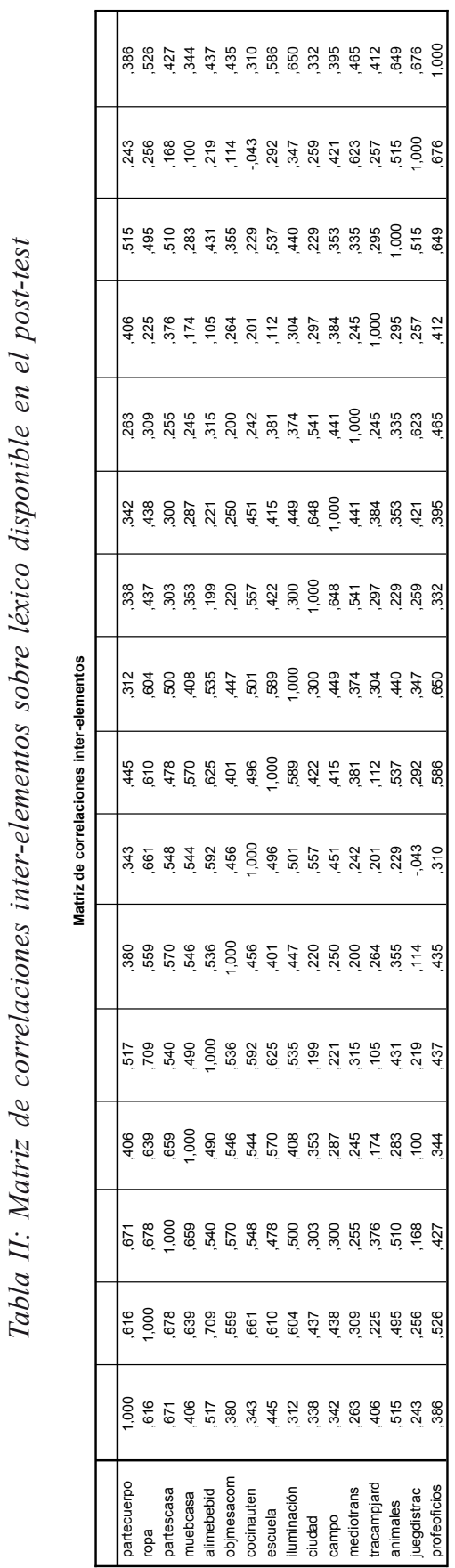


A continuación se ofrecen los estadísticos descriptivos realizados sobre el léxico disponible de los sujetos de la muestra de nuestra investigación. Se realizaron sobre cada uno de los dieciséis campos semánticos propuestos, pero aquí solo detallamos los datos totales sobre esta variable general para no excedernos de los límites de este trabajo.

En resumen, el grupo experimental obtuvo en el pre-test una media en el total del léxico disponible de 157,64; la desviación típica es de 80,93; y el valor de la curtosis es negativo, $-0,03$; con un coeficiente de asimetría positivo, 0,62 ; de valor mínimo 22 y máximo 347; y una amplitud intercuartil de 124.

En el grupo control la media de palabras disponibles es de 173,33; la desviación típica es 43,48 ; la curtosis es negativa al $-0,44$; el coeficiente de asimetría es también positivo 0,45; el valor mínimo es 113 y el máximo 262; y la amplitud intercuartil es de 67 .

La comparación de las medidas de tendencia central más significativas se resume en la Tabla I y a continuación los diagramas de cajas correspondientes se detallan en la Figura 1 de este trabajo, el cual nos indican que a pesar de que el grupo experimental en los campos semánticos parciales obtiene peores resultados, como veremos más adelante, en el léxico disponible global los resultados no fueron tan malos. El apuntamiento de la curva es mayor que en el grupo control, siendo la curtosis del primero más simétrica, con lo que los resultados podemos decir que son positivos.

Tabla III: Comparación de medidas de tendencia central sobre léxico disponible total entre los grupos experimental y control en el pre-test.

\begin{tabular}{|c|c|c|c|c|c|c|}
\hline TTLEXDISP & $\mathrm{N}$ & MEDIA & DESV. TÍP. & MEDIANA & CURTOSIS & ASIMETRÍA \\
\hline EXPERIMENTAL & 25 & 157,64 & 80,93 & 135 &,- 03 &, 62 \\
\hline CONTROL & 15 & 173,33 & 43,48 & 161 &,- 44 &, 45 \\
\hline
\end{tabular}

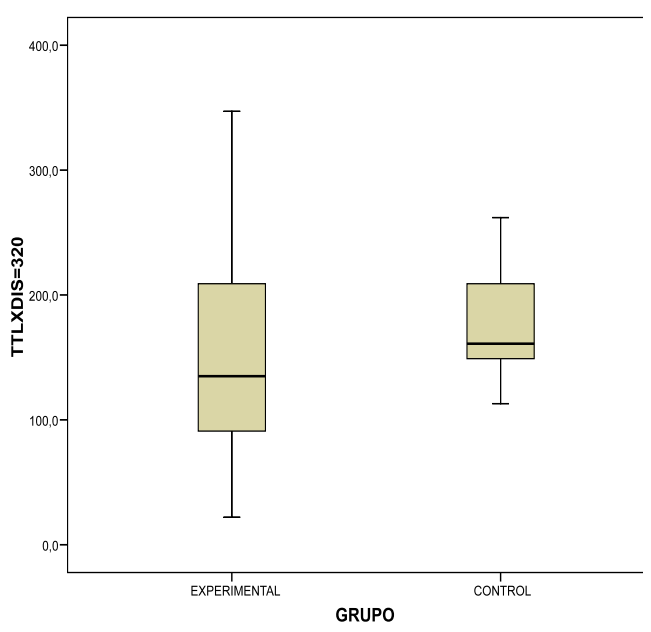

Figura 1: Histogramas sobre los datos totales del léxico disponible de los grupos experimental y control en el pre-test. 
En cuanto a los datos totales sobre léxico disponible en el post-test podemos decir, en resumen, que el grupo experimental obtuvo una media en el total del léxico disponible de 209,08; la desviación típica es de 71,78; y el valor de la curtosis 2,20, con un coeficiente de asimetría positivo de 0,12 , de valor mínimo 22 y máximo 397, y una amplitud intercuartil de 70 .

En el grupo control la media de palabras disponibles es de 209,03; la desviación típica es 70,20; la curtosis tiene valor negativo al $-0,32$; el coeficiente de asimetría es, sin embargo, positivo 0,52, el valor mínimo es 99 y el máximo 376; y la amplitud intercuartil es de 93.

La comparación de las medidas de tendencia central más significativas la ofrecemos de forma resumida en la Tabla IV; y la figura o diagrama de cajas correspondiente a esta variable en la Figura 2 de este trabajo.

Tabla IV: Comparación de medidas de tendencia central sobre léxico disponible total entre los grupos experimental y control en el post-test.

\begin{tabular}{|l|l|l|c|c|c|c|}
\hline TOTLEXDISP & $\mathrm{N}$ & MEDIA & DESV. TÍP. & MEDIANA & CURTOSIS & ASIMETRÍA \\
\hline EXPERIMENTAL & 25 & 209,08 & 71,78 & 202 & 2,20 &, 12 \\
\hline CONTROL & 27 & 209,03 & 70,20 & 200 &,- 32 &, 52 \\
\hline
\end{tabular}

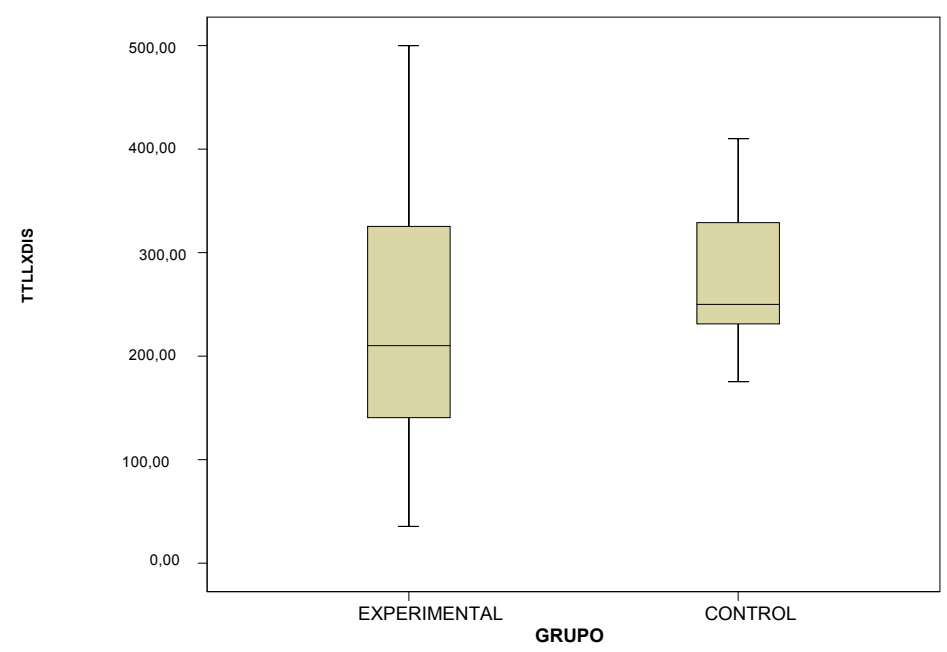

Figura 2. Histogramas sobre datos totales del léxico disponible de los grupos experimental y control en el post-test.

El grupo experimental consigue igualar al grupo control en el total del léxico disponible, como se observa en el diagrama de cajas de esta variable, la campana de Gauss del primero no es tan perfecta como en el segundo, pero la aproximación es bastante aceptable. 
Por último, ofrecemos dos figuras más que resumen la evolución positiva a favor del grupo experimental en la media de los dieciséis campos semánticos de su léxico disponible. Esto nos indica que este grupo ha mostrado una evolución constante mientras que el grupo control se ha mantenido en el mejor de los casos y ha empeorado en otros, como se observa en las figuras 3 y 4, donde señalamos además la media en los dieciséis campos semánticos del léxico disponible de ambos grupos en el pre-test y en el post-test.

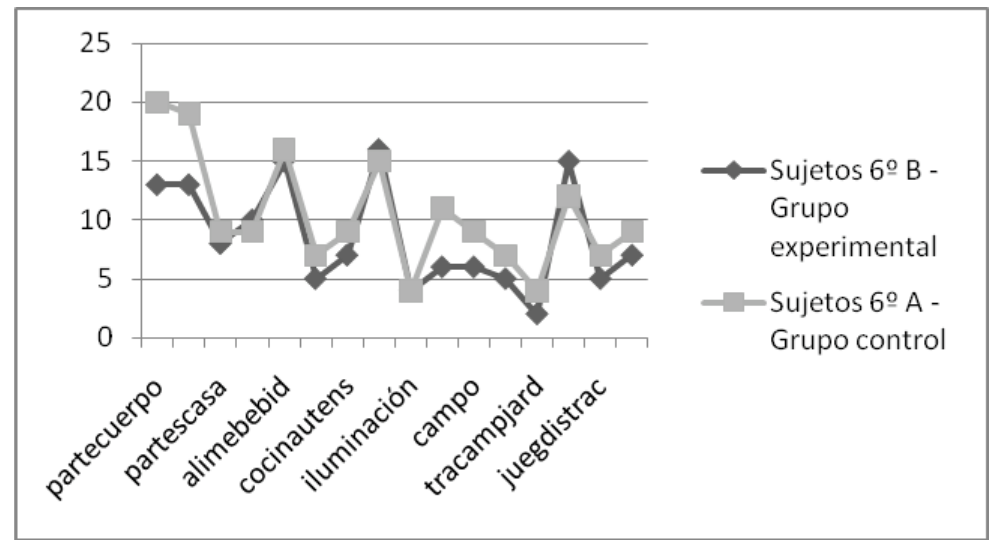

Figura 3. Media de léxico disponible por campos semánticos de los grupos experimental y control en el pre-test.

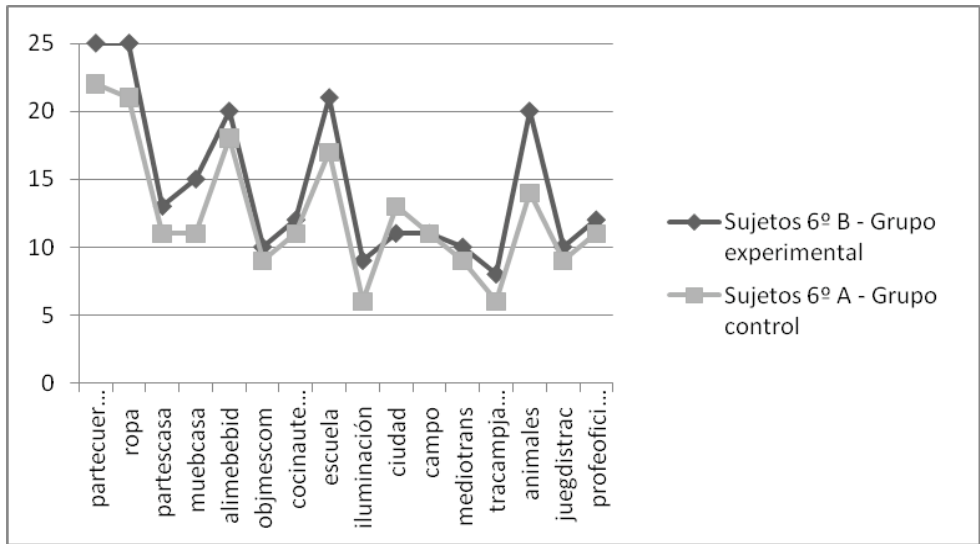

Figura 4. Media de léxico disponible por campos semánticos de los grupos experimental y control en el post-test.

En el total del léxico disponible, el rango promedio del grupo experimental demuestra que ha crecido notablemente con respecto al grupo control, que incluso llega a disminuir, como puede verse en la Tabla V. 
Tabla V: Rangos de la comparación de resultados sobre la variable léxico disponible: total léxico disponible entre el pre-test y post-test de los grupos experimental y control

\begin{tabular}{|l|l|l|l|c|c|}
\hline & GRUPO & \multicolumn{2}{|c|}{ N } & RANGO PROMEDIO & SUMA DE RANGOS \\
\hline \multirow{4}{*}{ TOTLEXDIS } & \multirow{2}{*}{ PRE-TEST } & EXPERIMENTAL & 26 & 24,50 & 637 \\
\cline { 3 - 6 } & \multirow{2}{*}{} & CONTROL & 26 & 28,50 & 741 \\
\cline { 2 - 6 } & \multirow{2}{*}{ POS-TEST } & EXPERIMENTAL & 25 & 27,26 & 696,50 \\
\cline { 3 - 6 } & & CONTROL & 27 & 25,80 & 681,50 \\
\hline
\end{tabular}

La figura 5 muestra esta evolución a favor del grupo experimental, y vemos cómo la suma de rangos de ambos grupos llega a igualarse y solaparse en el post-test, acortándose las grandes diferencias que se observaban al principio de esta investigación.

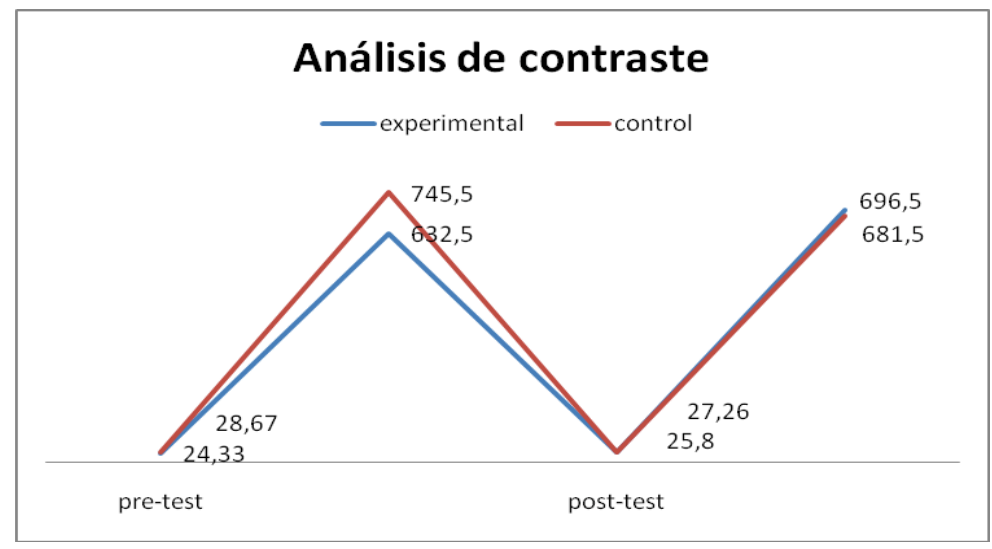

Figura 5. Análisis de contraste de la variable sobre léxico disponible: total léxico disponible entre el pre-test y post-test de los grupos experimental y control.

Para comprobar si el estadístico $U$ es significativo se utiliza el contraste $Z$ porque ha habido algunas puntuaciones iguales (empates). Por ejemplo, en "partes del cuerpo" $Z=$ $-3,12 p \leq 0,00$. Aquí se comprueba entonces si existen diferencias significativas entre los dos grupos de sujetos. Al ser muestras relacionadas el otro estadístico que se ofrece para ver si existen diferencias en el pre-test y post-test del grupo experimental, por un lado, y del grupo control, por otro es la $W$ de Wilcoxon, $W=518,50(19,94 * 26)$. El estadístico de $U$ de Mann-Whitney es igual a 167,50 en este ejemplo.

Los resultados de la Tabla IV muestran cómo de no haber diferencias significativas al 0,005 en los distintos campos sobre la variable léxico disponible en el pre-test, en el posttest se igualan aún más estas pequeñas diferencias, con lo que siguen sin ser significativas. Esto quiere decir que si había algunas diferencias entre los dos grupos al principio, éstas se atenúan y ambos grupos tienden a igualarse después del programa de intervención que constituye la parte central de esta investigación. 
Tabla VI: Estadísticos de contraste sobre la variable léxico disponible entre el pre-test y post-test.

\begin{tabular}{|c|c|c|c|c|c|}
\hline VARIABLE & & $\begin{array}{l}\text { U DE MANN- } \\
\text { WHITNEY }\end{array}$ & $\begin{array}{c}\text { W DE } \\
\text { WILCOXON }\end{array}$ & z & $\begin{array}{l}\text { SIG. ASINTÓT } \\
\text { (BILATERAL) }\end{array}$ \\
\hline \multirow{2}{*}{ PARTECUERPO } & PRE-TEST & 167,50 & 518,50 & $-3,12$ &, 00 \\
\hline & POS-TEST & 424 & 1909 & $-2,65$ &, 00 \\
\hline \multirow{2}{*}{$R O P A$} & PRE-TEST & 281 & 632 & $-1,04$ &, 29 \\
\hline & POS-TEST & 666 & 2151 &,- 09 & ,92 \\
\hline \multirow{2}{*}{ PARTESCASA } & PRE-TEST & 263 & 614 & $-1,37$ &, 16 \\
\hline & POS-TEST & 595 & 920 &,- 84 &, 39 \\
\hline \multirow{2}{*}{ MUEBCASA } & PRE-TEST & 247 & 598 & $-1,67$ & ,09 \\
\hline & POS-TEST & 593 & 918 &,- 86 &, 38 \\
\hline \multirow{2}{*}{ ALIMEBEBID } & PRE-TEST & 288 & 639 &,- 91 &, 36 \\
\hline & POS-TEST & 535 & 2020 & $-1,47$ &, 13 \\
\hline \multirow{2}{*}{ OBJMESACOM } & PRE-TEST & 262 & 613 & $-1,39$ &, 16 \\
\hline & POS-TEST & 569 & 2054 & $-1,12$ &, 26 \\
\hline \multirow{2}{*}{ COCINAUTEN } & PRE-TEST & 318,50 & 669,50 &,- 35 &, 72 \\
\hline & POS-TEST & 620 & 945 &,- 58 &, 56 \\
\hline \multirow{2}{*}{ ESCUELA } & PRE-TEST & 302,50 & 653 &,- 65 &, 51 \\
\hline & POS-TEST & 557 & 2042 & $-1,24$ &, 21 \\
\hline \multirow{2}{*}{ ILUMINACIÓN } & PRE-TEST & 327 & 678 &,- 20 &, 84 \\
\hline & POS-TEST & 653 & 978 &,- 23 &, 81 \\
\hline \multirow{2}{*}{ CIUDAD } & PRE-TEST & 269,50 & 620,50 & $-1,25$ &, 20 \\
\hline & POS-TEST & 644 & 2129 &,- 32 &, 74 \\
\hline \multirow{2}{*}{ САMPO } & PRE-TEST & 323 & 674 &,- 27 &, 78 \\
\hline & POS-TEST & 619 & 944 &,- 59 &, 55 \\
\hline \multirow{2}{*}{ MEDIOTRANS } & PRE-TEST & 267,50 & 618,50 & $-1,29$ &, 19 \\
\hline & POS-TEST & 553 & 878 & $-1,29$ &, 19 \\
\hline \multirow{2}{*}{ TRACAMPJARD } & PRE-TEST & 256 & 607 & $-1,52$ &, 12 \\
\hline & POS-TEST & 487 & 812 & $-2,01$ &, 04 \\
\hline \multirow{2}{*}{ ANIMALES } & PRE-TEST & 321 & 672 &,- 31 &, 75 \\
\hline & POS-TEST & 645 & 2130 &,- 31 &, 75 \\
\hline \multirow{2}{*}{ JUEGDISTRAC } & PRE-TEST & 317 & 668 &,- 38 &, 69 \\
\hline & POS-TEST & 601 & 926 &,- 78 &, 43 \\
\hline \multirow{2}{*}{ PROFEOFICIOS } & PRE-TEST & 326,50 & 677,50 &,- 21 &, 83 \\
\hline & POS-TEST & 656 & 2141 &,- 20 &, 84 \\
\hline \multirow{2}{*}{$\begin{array}{l}\text { TOTAL LÉXICO } \\
\text { DISPONIBLE }\end{array}$} & PRE-TEST & 286 & 637 &,- 95 &, 34 \\
\hline & POS-TEST & 637 & 2122 &,- 40 &, 68 \\
\hline
\end{tabular}




\section{Discusión y CONCLUSIONES}

Teniendo en cuenta los resultados obtenidos en el estudio estadístico intergrupos, o sea, el que compara los rendimientos del léxico disponible del grupo experimental y del grupo control, tanto en el pre-test como en el post-test, podemos afirmar que:

Los alumnos del grupo experimental mejoran de forma estadísticamente significativa su dominio de los siguientes campos semánticos: "partes del cuerpo", "ropa", "partes de la casa", "muebles de la casa", "cocina y sus utensilios", "escuela", "campo", "trabajos del campo y del jardín", "animales", "juegos y distracciones" y "profesiones y oficios" de su léxico disponible.

Los alumnos de sexto de Educación Primaria que siguen el Programa de intervención didáctica para el desarrollo de la competencia léxica (Pedelex) mejoran su léxico disponible, en el caso de los campos semánticos "iluminación y calefacción” y "medios de transporte", aunque esta optimización no se produce de manera estadísticamente significativa. Finalmente, los campos semánticos que quedaron sin confirmar estadísticamente son sólo tres: "objetos colocados en la mesa para la comida", "ciudad" y "medios de transporte".

A modo de síntesis, podemos decir que tomando como referencia la finalidad inicial de la investigación, es decir, la evaluación de los principales procesos implicados en la adquisición del léxico disponible, la utilidad del procedimiento aplicado consiste en obtener una puntuación que informe sobre la cantidad de léxico disponible de los sujetos experimentales.

Dado que nuestro estudio se ubica en el campo científico de la Didáctica de la Lengua, estos datos afirman que urge proponer e implementar planteamientos rigurosos e innovadores en las aulas de la educación obligatoria del sistema español, para ajustar la intervención pedagógica en contextos multiculturales, y de modo que el componente léxico no sea un elemento aislado en la enseñanza de las lenguas, sino una plataforma cognitiva que contribuya decididamente al desarrollo de la competencia comunicativa global de los sujetos cuya lengua materna es diferente al español.

El cambio por el que abogamos con esta experiencia debe afectar a la concepción global del área de Lengua castellana y Literatura en la Educación Obligatoria y al tratamiento del español como segunda lengua en este contexto. Por eso, opinamos que es necesaria la incorporación de las aportaciones de las nuevas ciencias del lenguaje y de lo regulado en el marco legal vigente, que es el que ofrece la posibilidad de hacer real esta línea de actuación.

Desde el punto de vista de la comunicación, parece conveniente insistir en las dificultades para observar la evolución de la competencia léxica en periodos relativamente cortos de tiempo como, en este caso, un curso escolar. Desde el punto de vista de la metodología utilizada en la parte empírica, esta investigación ha tomado como base un diseño cuasiexperimental, como se ha dicho ya, por la falta de aleatorización en la selección de la muestra; por tanto, las intervenciones didácticas llevadas a cabo se han realizado con unos estudiantes concretos y en unas aulas determinadas. Así, teniendo en cuenta este contexto escolar concreto - perteneciente a los alumnos de sexto curso de Educación Primaria-, los resultados obtenidos hay que entenderlos referidos sólo a dicho contexto y periodo evolutivo de los sujetos, o a contextos y edades semejantes, siempre que sean iguales en las variables intervinientes que se han considerado para la muestra en cuestión.

Por último, pese a que el contexto de la investigación es un aula real previamente configurada, según criteros del propio centro en los que no hemos intervenido, lo cierto es 
que la presencia del investigador y su actividad con los sujetos supone una modificación de las condiciones del estudio que no es posible eliminar y cuyo impacto es difícil de determinar.

Presumimos que este trabajo podría facilitar nuevos estudios que completaran el que aquí se presenta, pudieran ampliar algunos puntos del mismo o reproducir su esquema investigador aplicándolo a otros contextos sociales o lingüísticos, a sujetos de otras edades o a otro nivel educativo, siempre que se hagan las modificaciones pertinentes en cada caso. O cabría también la posibilidad de iniciar un trabajo que contemplase la competencia léxica como parte de la competencia discursiva global y se centrara en interacciones entre la lengua oral y la escrita en el proceso de desarrollo lingüístico y comunicativo.

En cualquier caso, la investigación aquí expuesta aspira a ser valorada como un logro positivo, como difusión de riquezas culturales y sociales para hacer avanzar en la educación lingüística precisamente en la línea en que las actuales sociedades plurales e interculturales lo requieren.

\section{REFERENCIAS BIBLIOGRÁFICAS Y WEBGRAFÍA}

Camacho, J. (2002). Estadística con SPSS para Windows (Versión 11). Madrid: Ra-Ma, 125296.

Dendaluce, I. (1989). Aspectos Metodológicos de la Investigación Educativa. Madrid: Narcea.

Fernández, G., Rico, A. M. ${ }^{\mathrm{a}}$, Molina, M J J., y Jiménez, Ma A. (2008). Léxico disponible de Melilla. Estudio sociolingüístico y repertorios léxicos. Madrid: Arco Libros.

García, M., Ibáñez, J., y Alvira, F. (1994). El análisis de la realidad social; métodos y técnicas de investigación. Madrid: Alianza Universidad.

Gougenheim, G. (1956). Le français élèmentaire. Louvain: Centre International de Dialectologie Générale.

Martín, M. (2004). La enseñanza del léxico español a través de Internet. Edinumen: Madrid.

Möller, J. (2001). Siglo XXI: ¿Innovamos? La enseñanza de una segunda lengua extranjera. Salobreña (Granada): Editorial Alhulia.

Möller, J., y Burbat, R. (2007). Collage 1A: Manual de enseñanza del alemán: Lektion 1-5. Granada: Universidad de Granada.

Müller, C. (1977). Principes et méthodes de statistique lexicale. Paris: Hachette.

Samper, M. (2002). Disponibilidad léxica en alumnos de español como lengua extranjera. Colección Monografías, $n^{\circ} 4$. Málaga: ASELE.

San Mateo, A. (2005). Aprendizaje del léxico español como lengua extranjera. Investigación sobre tres métodos. Madrid: UNED.

Ortega, L. y Iberri-Shea, G. (2005). "Longitudinal research in second language acquisition: Recent trends and future directions", in Annual Review of Applied Linguistics, vol. 25: 26-45.

Oster, U. (2009). "La adquisición de vocabulario en una lengua extranjera: de la teoría a la aplicación didáctica", en Porta Linguarum, 11: 33-50.

Prado, J. (2004). Didáctica de la Lengua y la Literatura para educar en el siglo XXI. Madrid: La Muralla, 308-309.

Sinclair, J. McH (2004). How to use corpora in Language Teaching. Amsterdam: John Benjamins Publishing.

Singleton, D. \& Ryan, L. (2004). Language Acquisition: The Age of Factor. Clevedon: Multilingual Matters. 
Vivanco, V. (2001). "La adquisición del vocabulario en una segunda lengua: estrategias cognitivas y lazos afectivos", en Revista de investigación e innovación en la clase de idiomas, 12: $177-188$.

VV. AA. (2006). Lengua. Deja huella. $6^{\circ}$ Primaria. Tercer Ciclo. Madrid: Anaya.

\section{Webgrafía}

Martín, S. (2009). "La revisión del concepto de vocabulario en la gramática de ELE", en Monográficos Marcoele, 9: 157-163. Didáctica del español como lengua Extranjera. Madrid: Expolingua. Disponible en Internet [en línea]: http://www.marcoele.com/descargas/ expolingua_1999.martin.pdf [Consulta del 17-07-2011]

Pérez, C. (2002). "Explotación de los corpora textuales informatizados para la creación de bases de datos terminológicas basadas en el conocimiento", Estudios de Lingüística Española (ELiEs), Universidad de Málaga. Disponible en Internet [en línea]: http://elies.rediris.es/ elies18/ [Consulta del 17-07-2011]

Real Academia Española (2004). Banco de datos (CREA). Corpus de referencia del español actual. Disponible en Internet [en línea]: http://www.rae.es/ [Consulta del 10-02-2010].

Sierra Martínez, G. (2005). Lingüística de corpus. Diseño y análisis de corpus textuales. Disponible en Internet: http://iling.torreingenieria.unam.mx/CursoCorpus2005/default.html [Consulta del 17-07-2011] 